\title{
Trial Paths Domain
}

National Cancer Institute

\section{Source}

National Cancer Institute. Trial Paths Domain. NCI Thesaurus. Code C95101.

A subject domain utilized for the submission of information encompassing and representing data, vocabulary or records related to trial paths. 